\title{
Predictive ultrasound factors of lymphatic invasion in rectal cancer: "extra-corporeal" study
}

\author{
José Manuel Ramírez-Rodríguez ${ }^{1}$, María José Palacios-Fanlo², Javier Ortego-Fernández-de-Retana ${ }^{3}$, \\ Vicente Aguilella-Diago ${ }^{1}$ and Mariano Martínez-Díez ${ }^{1}$ \\ ${ }^{1}$ Department of Surgery. Hospital Clínico Universitario de Zaragoza. Spain. ${ }^{2}$ Department of Surgery. \\ Hospital Nuestra Señora de Candelaria. Santa Cruz de Tenerife. Spain. ${ }^{3}$ Department of Pathology. Hospital Clínico \\ Universitario de Zaragoza. Zaragoza, Spain
}

\begin{abstract}
Objective: the accuracy of preoperative endorectal ultrasound in the status evaluation of lymph nodes is around $50-70 \%$, with a lack of eco-morphological patterns of clinical use. Since, accurate local staging is of great value in prognosis and decision-making we decided to analyze the referenced eco-morphological parameters in an attempt to find a proper predictive tool of clinical help that could improve the accuracy of rectal ultrasound.

Material and method: the resected specimens of 24 patients that were operated on by radical surgery because rectal cancer, without preoperative radiotherapy, were suspended in warm water and ultrasound scanned $\left(360^{\circ}\right.$ circular probe with a transducer of $10 \mathrm{MHz}$ ). All suspicious nodes were recorded and marked for the definitive histological report.

Results: from the 24 specimens, 318 nodes were imaged (210 benign and 100 involved). All ultrasound parameters analyzed were significant but only lobulation, echogenicity and hilar reflection were independent values. An score system was design with the addition of all parameters that showed a sensitivity of $98 \%$ and specificity of $99,1 \%$.

Conclusions: our study shows that a careful study of ultrasound lymph node images can get a high level of accuracy and better help in tailoring the treatment of any particular case.
\end{abstract}

Key words: Rectal cancer. Endorectal ultrasound. Lymph node.

Ramírez-Rodríguez JM, Palacios-Fanlo MJ, Ortego-Fernándezde-Retana J, Aguilella-Diago V, Martínez-Díez M. Predictive ultrasound factors of lymphatic invasion in rectal cancer: "extracorporeal” study. Rev Esp Enferm Dig 2011; 103: 299-303.

Financial support: This study was made possible by the Fundación Española de Patología Digestiva (Spanish Foundation for Digestive Pathology.

Received: 13-09-10.

Accepted: 02-02-11.

Correspondence: José M. Ramírez Rodríguez. Servicio de Cirugía. Hospital Clínico Universitario. C/ San Juan Bosco, 15. 50009 Zaragoza. Spain. e-mail: jramirez@unizar.es

\section{INTRODUCTION}

Colo-rectal carcinoma is the most common malign digestive neoplasm in countries with comparable geographical and socio-economic conditions to ours. Half of these tumors are located in the rectum and the recto-sigmoid junction. Rectal cancer is understood at this time as a differentiated oncologic entity due to the peculiar characteristics of its diagnosis and treatment. In these last few years, a multi-disciplinary approach to this entity, with individualized treatment, has achieved substantial improvement in terms of local recurrence, quality of life and survival (1). In this sense, the optimal results of this treatment are directly connected to the precision with which the preoperative study is made (2).

In rectal cancer, this loco-regional preoperative evaluation refers us both to the state of the primary tumor as well as to the situation of the meso-rectal lymph nodes, which are important in the prognosis of the patient and are influential in the taking of decisions (3).

Although in the last few decades diagnostic imaging techniques have improved greatly -e.g. magnetic resonance (MR) or computerized tomography (CT)-, endorectal ultrasound (E-R U/S) continues to be the most effective loco-regional pre-operative evaluation for cancer of the rectum $(4,5)$. Even though this is the case, the efficiency of evaluating lymph nodes is between 50 and $70 \%$ in most studies $(6,7)$. Since it has great negative predictive value, these results are due mostly to the inability of E-R U/S to differentiate metastatic from inflammatory nodes. There are no generally accepted echo-morphological patterns for lymphatic invasion and those which do exist are difficult to put into practice in clinical conditions.

Because of all this, we set out to analyze the known morphological parameters in order to find a clinically helpful tool that would improve diagnostic precision in evaluating meso-rectal lymph nodes. 
Table I. Echographic parameters analysed. Criteria for corresponding classification from 1 to 15

\begin{tabular}{|c|c|c|c|c|c|}
\hline & 1 & 2 & 3 & 4 & 5 \\
\hline Roundness Index & $4: 1$ & $3: 1$ & $2: 1$ & $4: 3$ & $1: 1$ \\
\hline Lobulation & None & Doubtful & Single & Double & Multiple \\
\hline Echogenicity & Isoechoic & Hipoechoic & $=$ Tumour & Hyper-echoic & Anechoic \\
\hline Inhomogeneity & No reflections & Fine & Individual area & Multiple fine & Multiple strong \\
\hline \multicolumn{6}{|l|}{ Delimited edge } \\
\hline (\% of the perimeter) & 5 & 25 & 50 & 75 & 100 \\
\hline \multicolumn{6}{|l|}{ Hypo-echoic halo } \\
\hline (\% of the perimeter) & 5 & 25 & 50 & 75 & 100 \\
\hline \multicolumn{6}{|l|}{ Perinodal halo } \\
\hline (\% of the perimeter) & 5 & 25 & 50 & 75 & 100 \\
\hline Hilar reflection & Single, not central & Single central & Some fine & Multiple fine & Multiple strong \\
\hline
\end{tabular}

\section{MATERIAL AND METHOD}

This study was approved by the Research Committee of our Hospital and was undertaken within a study for improvements in preoperative diagnosis of patients with rectal cancer, initiated by the Fundación Española de Patología Digestiva (Spanish Foundation for Digestive Pathology).

The surgical pieces of 24 patients -all subjected to previous resection or abdominal amputation due to rectal cancer, with total excision technique of the meso-rectum and without previous radiotherapy- were the basis for this study.

Immediately after surgery, the resected piece was submerged in warm water within a transparent methacrylate box specially designed for this study. The pieces remained suspended, fixed to an upper support through which the ultrasound probe was inserted. Thus prepared, they were studied echographically with an 1850 rotating probe of $360^{\circ}$ and $10 \mathrm{MHz}$ transducer mounted on a BK machine, model 2003 (BK Medica, Denmark).

In all the lesions, penetration of the rectal wall was evaluated according to Hildebrandt Classification (9). In addition, for the study of the node, Beynon's criteria were used (10) and every nodular image was considered to be a lymph node (hypo-, iso- or hyperechoic) in the peri-rectal fat. The whole echographic study was exhaustively documented and recorded for posterior analysis.

Each visualized node was marked by the insertion of a hooked needle duly labeled. After finishing the exploration, the piece was put in formaldehyde and sent to the Department of Pathology for final study. All the pieces were valuated by the same pathologist who was at no time informed of the echographic findings.

The ultrasound images obtained were analyzed in the Digital Treatment Centre of the Research Assistance Services at our University. The study of the images was made with no knowledge of the anatomo-pathological re- sults. After analyzing the bibliography (11-14) and in accordance with the study's purpose, in addition to the maximum and minimum diameter in each ganglion, we analyzed 8 morphological data cited as significant discriminators of tumor invasion (Table I).

The roundness index: which relates the maximum and minimum diameter of the node. Also, the existence or not of indentations, or lobulation; then echogenicity, understood as the difference in grey scale with respect to the tumor; the internal echographic pattern of the node, or inhomogeneity; the clearness of vision of the external margin, or delimitation; the existence of internal echoes near to the hilum, or hilar reflection; the sighting of an internal hypo-echoic ring or hypo-echoic halo and lastly, the existence of a hyper-echoic perinodal halo.

For the adequate analysis and statistical study of each parameter, scores were assigned according to the Hulsmann criteria (13) (Table I). The SPSS Programme (SPSS Inc. IL, USA) was used and the bivariate analysis was calculated by Student's t-test and the Mann-Whitney U test. The chosen confidence interval was $95 \%$ and the level of significance was placed at $\mathrm{p}<0.05$. The independent value of each parameter was made via logistic regression analysis, developing a probabilistic model as was the case with the calculation of sensitivity and specificity.

\section{RESULTS}

The "in vitro" study of the 24 resected pieces led to the identification and ultrasound-controlled marking of a total of 318 nodes, of which -on checking the anatomopathological study- 218 were found to be of an inflammatory nature and 100 were neoplastic.

The visualized nodes had an average maximum diameter of $5.8( \pm 3.02) \mathrm{mm}$, where the average for neoplasies was $7.4( \pm 2.9) \mathrm{mm}$ and for the non neo-plasies $4.6( \pm 1.7)$ $\mathrm{mm}(\mathrm{p}<0.001)$. 
Table II. Echographic parameters analysed. Results of the inferential bivariate statistical study. Typical deviation in parentheses

\begin{tabular}{llll}
\hline & $\begin{array}{l}\text { Non-neoplastic } \\
n=218\end{array}$ & $\begin{array}{l}\text { Neoplastic } \\
n=100\end{array}$ & $p$ \\
\hline Roundness Index & $3.13(0.78)$ & $4.19(0.79)$ & 0.05 \\
Lobulation & $1.78(0.93)$ & $3.92(0.98)$ & 0.001 \\
Echogenicity & $2(0.65)$ & $4.10(0.63)$ & 0.05 \\
Inhomogeneity & $2.05(0.71)$ & $3.75(1.20)$ & 0.001 \\
Delimited edge & $1.69(0.87)$ & $3.84(0.93)$ & 0.05 \\
Hypo-echoic halo & $1.33(0,73)$ & $2.32(0.95)$ & 0.001 \\
Peri-nodal halo & $1.77(0.89)$ & $3.02(0.90)$ & 0.001 \\
Hilar reflection & $2.17(0.95)$ & $4.26(0.85)$ & 0.001 \\
& & & \\
\hline
\end{tabular}

Concerning the statistical results of the individual analysis of each parameter based on the quantitative variable (Table II), we can observe that they are all significant $(\mathrm{p}<0.05)$. If we add all these values and turn this sum into a new variable, its average was 15.9 in non-neoplastic nodes and 29.4 in the neoplastic ones, this variable was also significant.

The neoplastic nodes were rounder $(79 \%$ showed a Roundness Index of 4 or 5 and this index was only found in $30 \%$ of negative nodes), with multiple lobulations (64\% compared with $5 \%$ of those not invaded), hypoechoic ( $87 \%$ of the neoplastic ones were hypo-echoic or anechoic and only $2 \%$ of the negatives), of a heterogenous pattern internally (multiple inhomogeneity in 64\% of cases as compared with 5\%), defined borders in more than $75 \%$ of their perimeter ( $66 \%$ of the positive nodes presented this pattern compared with only $2.3 \%$ of negative nodes), with strong internal echoes $(90 \%$ of the nodes scored 4 or 5 in this parameter), absence of hypoechoic halo (less than $15 \%$ presented this in more than $50 \%$ of their perimeter). And with perinodal hyper-echoic halo (which occupied more than $50 \%$ of the perimeter) in $72 \%$ of cases compared with only $18 \%$ when the node was negative. Figure 1 shows a graph representing these patterns.

After logistic regression analysis, lobulation, echogenicity and hilar reflection were the only parameters to show individual discriminatory significance of neoplastic lymphatic invasion (Table III). We see that according to a specific model, the increase in value of echogenicity increases the risk of the node being positive in almost 30 cases.

The assessment of diagnostic efficiency of the logistic regression analysis showed a sensitivity of $99 \%$ and a specificity of $99.1 \%$, with a positive predictive value (PPV) of $98 \%$ and a negative predictive value (NPV) of $99.5 \%$

Using the sum of all the morphological parameters, we were able to design a diagnostic "score", in such a way that if we consider 25 to be the cutting-off point above

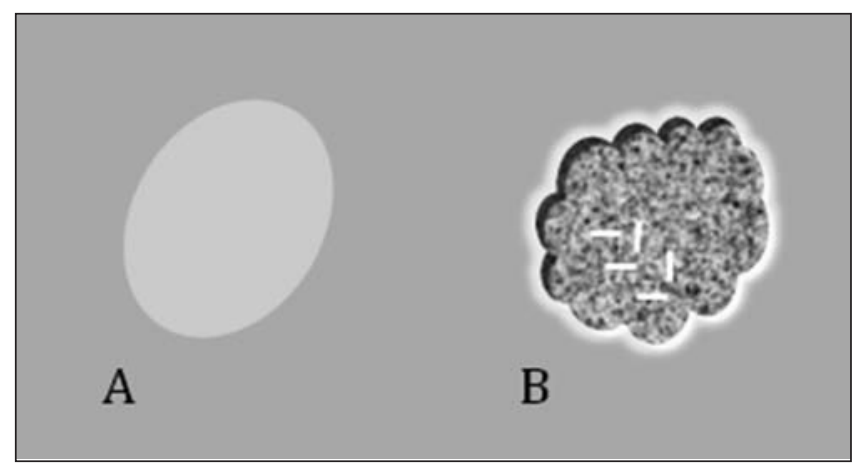

Fig. 1. Graphic representation of the echographic parameters for benignity and malignity. A. Un-invaded node. B. Pattern of malignity.

which the node should be considered to be neoplastic, sensitivity was $98 \%$ and specificity $99.1 \%(\mathrm{PPV}=98 \%$ and NPV $=99 \%)$.

\section{DISCUSSION}

The introduction of neoadjuvant therapy to the multimodal treatment of advanced rectal cancer has meant that the sphincter can be preserved in a greater number of patients, and that the number of local recurrences has decreased while survival rates have increased $(1,15)$. On the other hand, local excision is also now an alternative to radical surgery in early lesions (16).

The decision to give patients neoadjuvant therapy or local surgery requires the greatest preoperative diagnostic precision possible. In this sense, knowledge of the regional lymphatic situation in rectal tumors does not only determine the prognosis of the illness but is also a direct influence in the taking of decisions.

In spite of the latest advances and technological development, it is not possible to specify exactly the affection of the lymph nodes before surgical resection and their posterior histological examination. In fact, in cases where neoadjuvant therapy is offered, this nodular affection can never be exactly known and recognized; this is due to the alteration and destruction of tissue produced by radiochemotherapy.

There are numerous studies comparing E-R U/S, abdomino-pelvic CT and MR in the study of rectal tumors and despite the use of endo-rectal techniques in MR,

\section{Table III. Significant echographic parameters after logistic regression analysis}

\begin{tabular}{llll}
\hline Risk factor & Odds-ratio & Cl & $p$ \\
\hline Lobulation & 7.990 & $2.365-26.987$ & 0.001 \\
Echogenicity & 29.535 & $5.520-158.021$ & 0.000 \\
Hilar reflection & 5.313 & $1.925-14.665$ & 0.001
\end{tabular}


which has led to a significant improvement in the precision of this technique $(17,18)$-and has moved it closer to the results obtained by E-R U/S- the latter is usually the first choice mainly because it is easy to use and innocuous. It is also the only technique which allows guided fine-needle cytological puncture to be performed $(6,19)$.

It is generally agreed that E-R U/S is better at excluding than confirming the illness. If no suspicious image of the lymph node is observed, this diagnosis can be better believed than when it says there is an invasion (6).

As was the case 20 years ago, most examiners continue to base their echographic prediction of lymph node invasion on the identification of circular or oval hypoechoic lesions in the meso-rectal zone $(10,11)$. This discriminatory simplicity has led to numerous diagnostic errors $(7,20)$, so that when there is a possibility of error in the form of false negatives (infra-staging), and the conviction that it is better to over-treat then under-treat a cancer patient, most echographers tend to declare all nodes detected during the exploration to be neoplastic.

Over time, ultrasound technology and the quality of the images have improved and new studies have appeared which establish new criteria for lymphatic invasion. So for Hildebrandt (21) the fundamental discriminatory echographic parameters were echogenicity and the size of the node (benign at $<5 \mathrm{~mm}$ ). In this sense, the size of the node is used as an individual invasion criterion but -although in our study this variable turned out to be significant- $18 \%$ of the nodes of less that $5 \mathrm{~mm}$ which were analyzed were metastatic. As for echogenicity, or the relation of grays with respect to a primary tumor, we have been able to confirm that hypo-echoic or anechoic nodes are highly suggestive of metastasis. Echogenicity also has great discriminatory value as an independent variable.

The other two parameters concerning the internal structure of the node and that behaved as independent discriminatory variables were lobulation and hilar reflection. Lobulation presents areas of irregularity of the margin which are very suggestive of malignity, as is the case with de-structured hilar reflection, i.e. rupture and alteration of the central echogenic line, typical of the benign node (22).

Most of the morphological parameters that were analyzed are easy to recognize by an experienced echographer, but are subject to a great amount of variety. For this reason, Hulsmann's work (13) is very important for transforming each variable into a points score (Table I), thereby lessening the subjectivity of the echographer. From a practical point of view, as we have found in our work, the use of this points score makes it possible to develop specific predictive mathematical models. The simplest and easiest to use, for us, was the sum of all the parameters.

We should underline the importance of technical development in these studies. The majority of previous studies were carried out with probes with a frequency of
5 or $7.5 \mathrm{MHz}$. As we now used a $10 \mathrm{MHz}$ probe, in theory we have benefited from higher resolution.

To sum up; ultrasound continues to advance (23) and the echographer who is interested in studying rectal tumors with dedication and interest should not only must know all the possibilities that the ultrasound has to offer, but also what exploration can achieve. Our study shows that a detailed analysis of the images of nodes can achieve very high diagnostic efficiency and improve the selection of patients.

\section{REFERENCES}

1. Lindsetmo RO, Joh YG, Delaney CP. Surgical treatment for rectal cancer: an international perspective on what the medical gastroenterologist needs to know. World J Gastroenterol 2008;14:3281-9.

2. Meredith KL, Hoffe SE, Shibata D. The multidisciplinary management of rectal cancer. Surg Clin North Am 2009;89:177-215.

3. Koh DM, Brown G, Husband JE. Nodal staging in rectal cancer. Abdom Imaging 2006;31:652-9.

4. Bipat S, Glas AS, Slors FJ, Zwinderman AH, Bossuyt PM, Stoker J. Rectal cancer. Local staging and assessment of lymph node involvement with endoluminal US, CT, and MR imaging -A meta-analysis. Radiology 2004;232:773-83.

5. Chun HK, Choi D, Kim MJ, Lee J, Yun SH, Kim SH, et al. Preoperative staging of rectal cancer: comparison of 3-T high-field MRI and endorectal sonography. AJR Am J Roentgenol 2006;187:1557-62.

6. Srinivas R, Puli SR, Reddy JB, Bechtold ML, Choudhary A, Antillon $\mathrm{MR}$, et al. Accuracy of endoscopic ultrasound to diagnose nodal invasion by rectal cancers: a meta-analysis and systematic review. Ann Surg Oncol 2009;16:1255-65.

7. Palacios Fanlo MJ, Ramirez Rodriguez JM, Aguilella Diago V, Arribas del Amo D, Martinez Diez M, Lozano Mantecon R. Ecografía endorrectal en los tumores de recto: eficacia, causas de error y limitaciones. Rev Esp Enferm Dig 2000;92:222-6.

8. Schaffzin DM, Wong WD. Endorectal ultrasound in the preoperative evaluation of rectal cancer. Clin Colorectal Cancer 2004;4:124-32.

9. Hildebrandt U, Feifel G. Preoperative staging of rectal cancer by intrarectal ultrasound. Dis Colon Rectum 1985;28:42-6.

10. Beynon J, Mortensen NJM, Foy DMA, Channer DL, Rigby H, Virjee J. Preoperative assessment of mesorectal lymph node involvement in rectal cancer. Br J Surg 1989;76:276-9.

11. Tio TL, Tygat NJ. Endoscopic ultrasonography in analysing periintestinal lymph node abnormality: preliminary results of studies in vitro and in vivo. Scand J Gastroenterol (suppl) 1986;21:15863.

12. Glaser F, Schlag P, Herfarth C. Endorectal ultrasonography for the assessment of invasion of rectal tumors and lymph node involvement. Br J Surg 1990;77:883-7.

13. Hulsmans FH, Bosma A, Mulder PJJ, Readers JWAJ, Tytgat GNJ. Perirectal lymph nodes in rectal cancer: in vitro correlation of sonographic parameters and histopathologic findings. Radiology 1992; 184:553-60.

14. Nielsen MB, Qvitzau S, Pedersen JF. Detection of pericolonic lymph nodes in patients with colorrectal cancer: an invitro and in vivo study of the efficacy of endosonography. AJR 1993;161:57-60.

15. Janjan NA, Crane C, Feig BW, Cleary K, Dubrow R, Curley S, et al. Improved overall survival among responders to preoperative chemoradiation for locally avanced rectal cancer. Am J Clin Oncol 2001; 24:107-12.

16. Ramirez JM, Aguilella V, Gracia JA, Ortego J, Escudero P, Valencia J, et al. Local full-thickness excision as first line treatment for sessile rectal adenomas: long-term results. Ann Surg 2009;249: 225-8.

17. Gualdi GF, Casciani E, Guadalaxara A, d'Orta C, Polettini E, Pappalardo G. Local staging of rectal cancer with transrectal ultrasound and endorectal magnetic resonance imaging: comparison with histologic findings. Dis Colon Rectum 2000;43:338-45. 
18. Bianchi PP, Ceriani C, Rottoli M, Torzilli G, Pompili G, Malesci A, et al. Endoscopic ultrasonography and magnetic resonance in preoperative staging of rectal cancer: comparison with histologic findings. J Gastrointest Surg 2005;9:1222-7.

19. Vilmann P. Endoscopic ultrasonography-guided fine-needle aspiration biopsy of lymph nodes. Gastrointest Endosc 1996;43:S2429.

20. Navarro A, Hoyuela C, Rius J, Pierres M, Rodríguez Santiago J, Veloso E, et al. Importancia de la ecografía endorrectal en el estudio de la neoplasia de recto. Correlación con los hallazgos histológicos. Cir Esp 1997;62:102-7.
21. Hildebrandt U, Klein T, Feifel G, Schwarz HP, Koch B, Schmitt RM. Endosonography of pararectal lymph nodes. In vitro and in vivo evaluation. Dis Colon Rectum 1990;33:863-8.

22. Rubaltelli L, Proto E, Salmaso R, Bortoletto P, Candiani F, Cagol P. Sonography of abnormal lymph nodes in vitro: correlation of sonographic and histologic findings.AJR Am J Roentgenol 1990;155: 1241-4.

23. Martínez-Ares D, Alonso-Aguirre PA, Souto-Ruzo J, Martín-Granizo-Barrenechea I, Pereira-Bueno S, Cid-Gómez L, et al. Ultrasonography is an accurate technique for the diagnosis of gastrointestinal tumors in patients without localizing symptoms. Rev Esp Enferm Dig 2009; 101:773-86. 